\title{
Displaced Object Recognition Memory in Rats
}

Ali N. Mclagan and Jena B. Hales*

Department of Psychological Sciences, University of San Diego, San Diego, CA, USA

*For correspondence: ihales@sandiego.edu

[Abstract] The Displaced Object Recognition (DOR) task, sometimes called the Novel Object Location task, assesses spatial recognition memory without navigational demands, explicit instruction, or the need for multiple days of training. This memory task has two phases. First, the subject is familiarized to an open arena with two objects and is allowed to explore the objects. Following a delay period, the subject returns to the arena, but one of the previous objects has been moved to a new location. Greater exploration of the displaced object is used as the index of memory for the previous object location. An advantage of the DOR task is that subjects can be tested without explicit training, since this task exploits the natural tendency to be more interested in something novel. The spontaneous aspect of this task allows for the testing of animals as well as human populations that are unable to follow verbal instructions, such as babies. Therefore, this powerful test of recognition memory can be administered similarly for many species, including rats and humans, allowing for better translatability.

Keywords: Hippocampus, Spatial memory, Novel object recognition, Rat, Behavior, Novel object location

[Background] Animal models are critical for developing a better understanding of human memory function and dysfunction. Early animal studies examining memory dysfunction reported the critical importance of the hippocampus and surrounding cortical regions within the medial temporal lobes (Winocur, 1990; Zola-Morgan and Squire, 1990; Squire et al., 2001). One area of memory function that has been found to be particularly dependent on the hippocampus is spatial memory formation and retrieval. Many of the spatial memory tasks which are most sensitive to hippocampal damage or disruption are those that involve navigation, such as the radial arm maze, Barnes maze, and Morris Watermaze (Clark et al., 2007). Although such tasks are valuable, there are some limitations and potential confounds in using tasks that require navigation. For example, certain populations may have difficulty in navigating, but can still form and retrieve spatial memories. In addition, the performance aspect of navigation, independent of the spatial memory, may require the recruitment of additional brain areas. Therefore, it is important to have tests of spatial memory that are non-navigational. The DOR task provides a means for assessing spatial memory recognition without the animal having to navigate to a learned reference location in order to express the memory. 


\section{Materials and Reagents}

Note: All materials and reagents listed are examples based on what has been used successfully using the protocol provided; however, using different bedding, objects, rat strains/ages could also be successful.

1. Bed-o-cob $1 / 4$ in (Fisher Scientific, Harlan Teklad, catalog number: NC9335529)

2. Screws

3. Wide-Mouth Opaque Amber Plastic bottles $250 \mathrm{ml}$ (Thermo Scientific, Nalgene, catalog number: 02-923-103)

4. Rats (any strain that is able to visually discriminate objects)

5. Ethanol 70\% (Fisher Scientific, Decon Laboratories, catalog number: 04-355-223)

\section{Equipment}

1. Apparatus: opaque storage bin, dimension $60.7 \mathrm{~cm}(\mathrm{~W}) \times 40.4 \mathrm{~cm}(\mathrm{D}) \times 41.9 \mathrm{~cm}(\mathrm{H})$ (Rubbermaid, 18 gal, 2215)

2. Drill (e.g., Black+Decker Cordless Power Drill)

3. Video camera (e.g., Sony Handycam camcorder, such as HDR-CX405)

4. Timer (e.g., Fisherbrand Digital Timer, catalog number: 06-662-55)

\section{Software}

Note: Software listed below describes what has been used successfully using the protocol provided; however, using alternative software that allows for scoring of object exploration could also be successful.

1. Analysis software (ODLog 2, macropod software, http://www.macropodsoftware.com/odlog/)

\section{Procedure}

Note: All experiments are performed during the light phase (between 09:00 and 16:00) under fluorescent overhead lighting.

1. Setting up the apparatus

Remove the bottle caps from the four bottles, and use the drill and screws to secure the caps to the bottom of the storage bin with the open side facing up. Place one cap in each of the four quadrants as shown in Figure $1 \mathrm{~A}$.

2. Handling and habituating the rats to the experimenter and testing apparatus For 2 days prior to testing, rats should be handled for 5 min each day by the experimenter. Each rat should also spend 5 min on each day in the empty apparatus (with bed-o-cobs covering the 
floor and four bottle caps) to habituate.

\section{A. Setup}

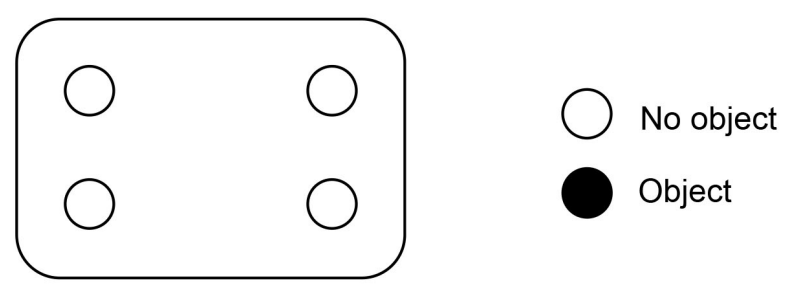

\section{B. Familiarization}

\section{Bottom-top}

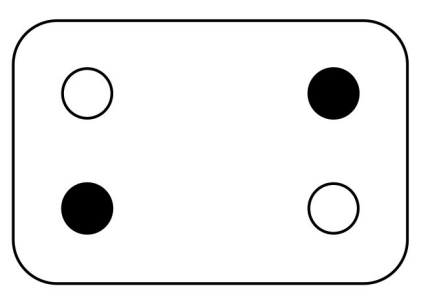

C. $\underline{\text { Test }}$

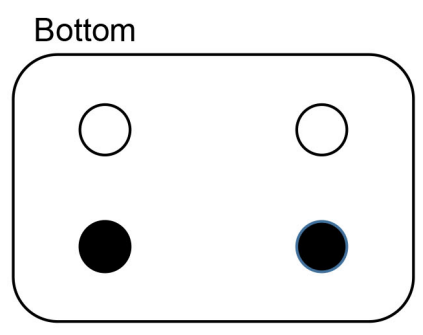

Top-bottom
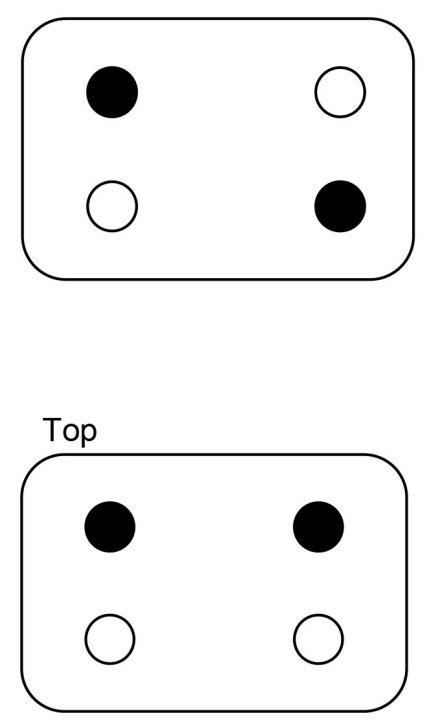

Figure 1. Experimental setup and object configurations for familiarization and testing. All diagrams are depicting the bird's eye view of the DOR apparatus.

3. Familiarization and delay period (Figure 2)

a. Turn on video camera positioned above testing apparatus.

b. Twist to secure bottles onto two of the caps in one of the familiarization configurations (Figure 1B). Be sure to counterbalance configurations across rats. Sprinkle bed-o-cobs around the bottom of the apparatus covering all of the bottle caps.

c. Start the video recording.

d. Hold testing sheet in front of camera listing the rat number and "familiarization".

e. Place the rat into the apparatus and start the timer for $15 \mathrm{~min}$.

f. After $15 \mathrm{~min}$, stop the recording, return the rat to the home cage, and write down the time on a testing sheet. The test time for the rat will be the current time $+3 \mathrm{~h}$ (delay).

g. Spray the stimuli with $70 \%$ ethanol and let them dry. Remove any feces from the apparatus.

h. Start the next rat using the same procedure (b-h above). 
4. Testing

a. When a rat is ready for testing ( $3 \mathrm{~h}$ after the familiarization phase), secure the bottles onto two of the caps in one of the test configurations (Figure 1C). Be sure to counterbalance configurations across rats. Make sure bed-o-cobs are still covering the bottle caps.

b. Start the video recording, and hold the testing sheet in front of the camera listing the rat number and "test".

c. Place the rat into the apparatus and start the timer for $15 \mathrm{~min}$.

d. After $15 \mathrm{~min}$, stop the recording, return the rat to the home cage, and spray the stimuli with $70 \%$ ethanol.

e. Continue this procedure (a-d) until all rats have been tested. Move the video files over to the computer, and turn off the camera.

f. Video 1 illustrates a typical DOR familiarization trial for two rats tested in adjacent testing bins.

Note: All testing intervals and timings are based on previous data using the Novel Object Recognition task. Familiarization and testing lengths of 15-min allow enough time for the animal to explore the objects. The three-hour delay interval has been used successfully to show a hippocampal-dependent memory effect using the Novel Object Recognition task previously (Broadbent et al., 2010). Shorter time delays may not show hippocampal dependency, and longer time delays can lead to weaker memories in control animals.

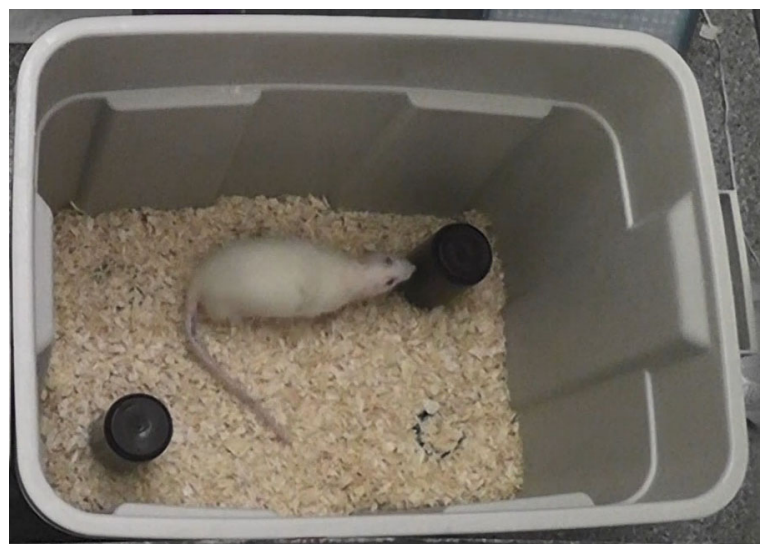

Figure 2. Familiarization Session. Photograph of a rat during familiarization. This behavior would be scored as "exploring the object", since its nose is pressed up to the object on the right.

\section{Data analysis}

Note: Use ODLog 2 software to analyze the video files.

1. Set up ODLog 2 so that the program stops scoring once it reaches a summed value of $30 \mathrm{~s}$ (as shown in Figure 3). 


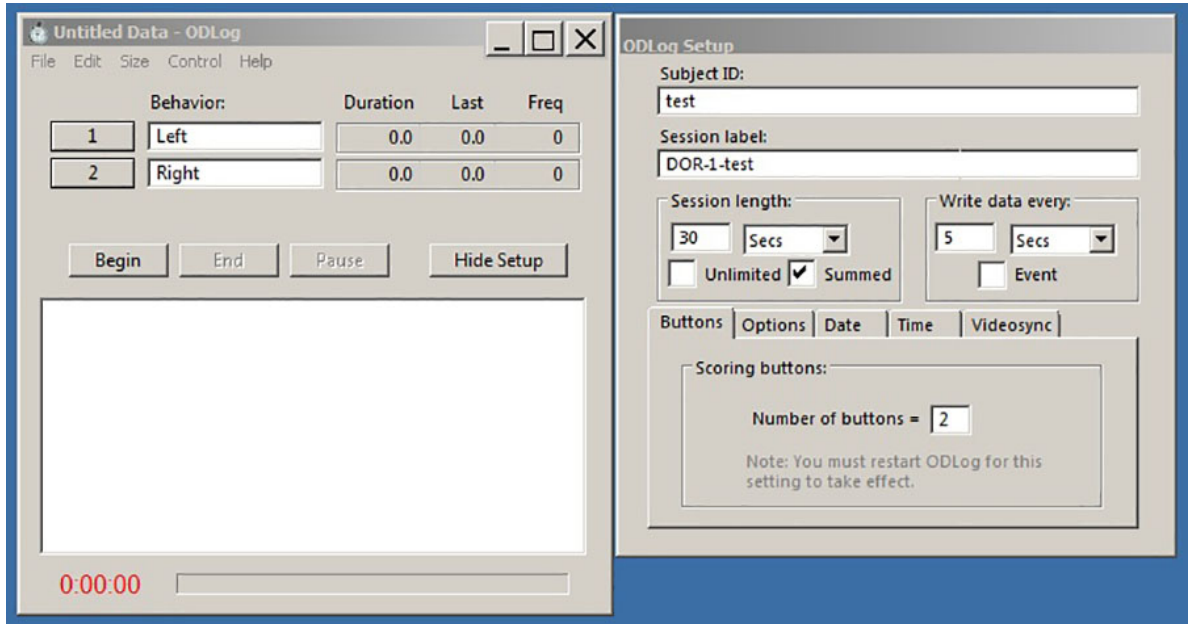

Figure 3. Program setup screen for ODLog 2 software. Once the program is open, the parameters should be set as shown above. The activated keys can be set from the Edit, Configure Keys drop down. Select "Sum" for keys 1 and 2.

2. Start the video file, and press "begin" on the ODLog 2 program as soon as the rat is placed into the apparatus. Place your left index finger on the left on key "1", which corresponds to the left object on the screen, and right index finger on the right on key "2", which corresponds to the right object on the screen. As soon as the rat places its nose up to an object to explore it (as shown in Figure 2), press down the corresponding key and keep it pressed down until the rat leaves the object or turns its head away, at which point, pick up your finger. It is important to be consistent in using the same scoring criteria for all rats.

3. Do not score if the rat is chewing the objects, climbing on the objects, or sniffing above, behind, or next to the objects.

4. Score the behavior until the rat reaches $30 \mathrm{~s}$ of total object exploration (the program will automatically stop once this summed value is reached). Save the summary document (yellow screen that pops up) and save the data file (txt document) before closing the program. Close the program after scoring each animal. A $30 \mathrm{~s}$ limit of total object exploration is used based on previous research using the Novel Object Recognition task, which showed the highest preference for the novel object to be within the first $30 \mathrm{~s}$ (after which, the novel object gains familiarity).

5. Open the program to score the next video file.

6. Enter data into an Excel spreadsheet with the following columns:
a. Subject \#
b. Group: this could be a within-subjects group or a between-subjects group label
c. Displaced Side $(L / R)$ : was the object on the left $(L)$ or the right $(R)$ displaced?
d. Time L: time spent exploring the object on the left
e. Time R: time spent exploring the object on the right
f. Time Displaced: time spent exploring the displaced object (equal to either Time $L$ or Time 
R)

g. Total Time: how long the rat was in the apparatus before reaching $30 \mathrm{~s}$ of object exploration

h. Total \% Displaced (Time Displaced/30* 100).

7. Calculate \% time exploring the displaced object by averaging the "Total \% Displaced" values for all rats within a group. Difference from chance (50\%) can be calculated using a one sample $t$ test, and group differences can be calculated with an independent (for between-subjects design) or paired sample $t$-test (for within-subjects design). Figure 4 depicts representative data obtained with this procedure.

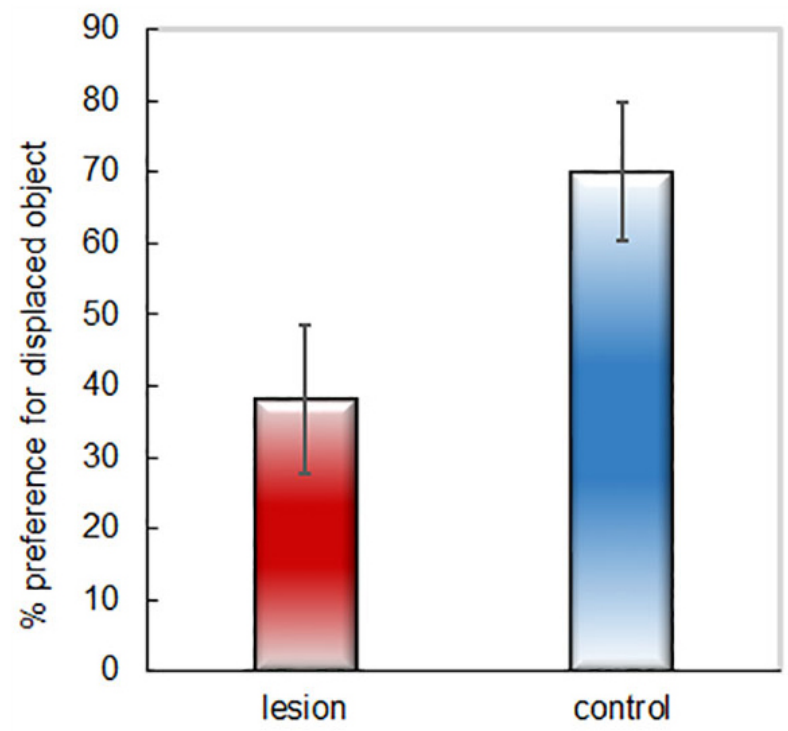

Figure 4. Example of representative data. Percent time spent exploring the displaced object during the $30 \mathrm{~s}$ of total object exploration for two groups of rats (lesion = excitotoxic hippocampal-lesioned rats; control = sham-lesion control rats).

\section{Acknowledgments}

This protocol was adapted from Hales et al., 2014. Recent work has been supported by the University of San Diego, College of Arts and Sciences. The authors thank Marta Collett for her insightful comments and feedback.

\section{Competing interests}

The authors have no conflicts of interest.

\section{Ethics}

All experimental procedures were approved by the Institutional Animal Care and Use Committees at the University of San Diego and at the University of California, San Diego. 


\section{References}

1. Broadbent, N. J., Gaskin, S., Squire, L. R., and Clark, R. E. (2010). Object recognition memory and the rodent hippocampus. Learn Mem 17: 5-11.

2. Clark, R. E., Broadbent, N. J. and Squire, L. R. (2007). The hippocampus and spatial memory: findings with a novel modification of the water maze. J Neurosci 27(25): 6647-6654.

3. Hales, J. B., Schlesiger, M. I., Leutgeb, J. K., Squire, L. R., Leutgeb, S. and Clark, R. E. (2014). Medial entorhinal cortex lesions only partially disrupt hippocampal place cells and hippocampusdependent place memory. Cell Rep 9(3): 893-901.

4. Squire, L. R., Clark, R. E. and Knowlton, B. J. (2001). Retrograde amnesia. Hippocampus 11(1): 50-55.

5. Winocur, G. (1990). Anterograde and retrograde amnesia in rats with dorsal hippocampal or dorsomedial thalamic lesions. Behav Brain Res 38(2): 145-154.

6. Zola-Morgan, S. M. and Squire, L. R. (1990). The primate hippocampal formation: evidence for a time-limited role in memory storage. Science 250(4978): 288-290. 